\title{
Nucleosynthesis in Black-Hole-Forming Supernovae
}

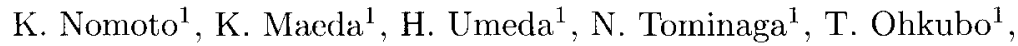 \\ J. Deng ${ }^{1}$, P. A. Mazzali ${ }^{1,2}$ \\ 1 Department of Astronomy \& Research Center for the Early Universe, \\ University of Tokyo, Tokyo, Japan; \\ nomoto@astron.s.u-tokyo.ac.jp \\ 2 Osservatorio Astronomico, Via Tiepolo, 11, 34131 Trieste, Italy
}

Summary. Stars more massive than $\sim 20-25 \mathrm{M}_{\odot}$ form a black hole at the end of their evolution. Stars with non-rotating black holes are likely to collapse "quietly" ejecting a small amount of heavy elements (faint supernovae). In contrast, stars with rotating black holes are likely to give rise to very energetic supernovae (hypernovae). We present distinct nucleosynthesis features of these two types of "black-hole-forming" supernovae. Nucleosynthesis in hypernovae is characterized by larger abundance ratios $(\mathrm{Zn}, \mathrm{Co}, \mathrm{V}, \mathrm{Ti}) / \mathrm{Fe}$ and smaller $(\mathrm{Mn}, \mathrm{Cr}) / \mathrm{Fe}$ than normal supernovae, which can explain the observed trend of these ratios in extremely metalpoor stars. Nucleosynthesis in faint supernovae is characterized by a large amount of fall-back. We show that the abundance pattern of the recently discovered most Fe-poor star, HE0107-5240, and other extremely metal-poor carbon-rich stars are in good accord with those of black-hole-forming supernovae, but not pair-instability supernovae. This suggests that black-hole-forming supernovae made important contributions to the early Galactic (and cosmic) chemical evolution. Finally we discuss the nature of first (Pop III) Stars.

\section{Hypernovae and Faint Supernovae}

Among the important developments in recent studies of core-collapse supernovae are the discoveries of two distinct types of supernovae (SNe) (Fig. 1) $[16,23]: 1)$ very energetic SNe (hypernovae), whose kinetic energy (KE) exceeds $10^{52} \mathrm{erg}$, about 10 times the KE of normal core-collapse SNe (hereafter $\left.E_{51}=E / 10^{51} \mathrm{erg}\right)$, and 2$)$ very faint and low energy SNe $\left(E_{51} \lesssim 0.5\right.$; faint supernovae). These two types of supernovae are likely to be black-hole-forming supernovae with rotating or non-rotating black holes. We compare their nucleosynthesis yields with the abundances of extremely metal-poor (EMP) stars to identify the Pop III (or first) supernovae. We show that the EMP stars, especially the C-rich class, are likely to be enriched by black-hole-forming supernovae. 


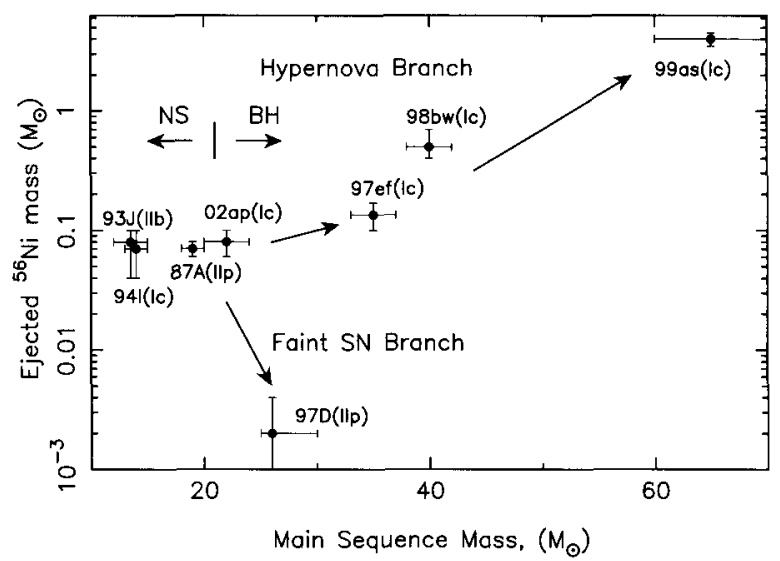

Fig. 1. The ejected ${ }^{56} \mathrm{Ni}$ mass as a function of the main sequence mass of the progenitors for several supernovae/hypernovae [16, 22, 23].

\section{Nucleosynthesis in Hypernova Explosions}

In core-collapse supernovae/hypernovae, stellar material undergoes shock heating and subsequent explosive nucleosynthesis. Iron-peak elements are produced in two distinct regions, which are characterized by the peak temperature, $T_{\text {peak }}$, of the shocked material. For $T_{\text {peak }}>5 \times 10^{9} \mathrm{~K}$, material undergoes complete Si burning whose products include $\mathrm{Co}, \mathrm{Zn}, \mathrm{V}$, and some Cr after radioactive decays. For $4 \times 10^{9} \mathrm{~K}<T_{\text {peak }}<5 \times 10^{9} \mathrm{~K}$, incomplete Si burning takes place and its after decay products include $\mathrm{Cr}$ and $\mathrm{Mn}[9,32]$.

\subsection{Supernovae vs. Hypernovae}

The right panel of Fig. 2 shows the composition in the ejecta of a $25 \mathrm{M}_{\odot}$ hypernova model $\left(E_{51}=10\right)$. The nucleosynthesis in a normal $25 \mathrm{M}_{\odot} \mathrm{SN}$ model $\left(E_{51}=1\right)$ is also shown for comparison in the left panel of Fig. 2 [33].

We note the following characteristics of nucleosynthesis with very large explosion energies $[19,20,21,25]$ :

1. Both complete and incomplete Si-burning regions shift outward in mass compared with normal supernovae, so that the mass ratio between the complete and incomplete Si-burning regions becomes larger. As a result, higher energy explosions tend to produce larger $[(\mathrm{Zn}, \mathrm{Co}, \mathrm{V}) / \mathrm{Fe}]$ and smaller $[(\mathrm{Mn}, \mathrm{Cr}) / \mathrm{Fe}]$, which can explain the trend observed in very metal-poor stars [36].

2. In the complete Si-burning region of hypernovae, elements produced by $\alpha$-rich freeze out are enhanced. Hence, elements synthesized through capturing of $\alpha$-particles, such as ${ }^{44} \mathrm{Ti},{ }^{48} \mathrm{Cr}$, and ${ }^{64} \mathrm{Ge}$ (decaying into ${ }^{44} \mathrm{Ca}$, ${ }^{48} \mathrm{Ti}$, and ${ }^{64} \mathrm{Zn}$, respectively) are more abundant. 

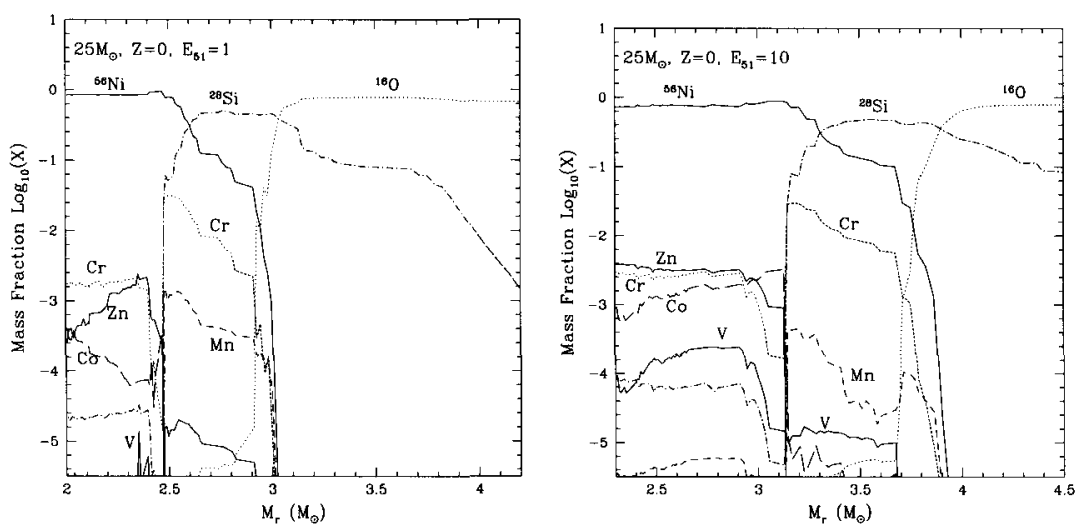

Fig. 2. Abundance distribution plotted against the enclosed mass $M_{r}$ after the explosion of Pop III $25 \mathrm{M}_{\odot}$ stars with $E_{51}=1$ (left) and $E_{51}=10$ (right) [33].

3. Oxygen burning takes place in more extended regions for the larger KE. Then more $\mathrm{O}, \mathrm{C}, \mathrm{Al}$ are burned to produce a larger amount of burning products such as $\mathrm{Si}, \mathrm{S}$, and Ar. Therefore, hypernova nucleosynthesis is characterized by large abundance ratios of $[\mathrm{Si}, \mathrm{S} / \mathrm{O}]$, which can explain the abundance feature of M82 [34].

\subsection{Hypernovae and $\mathrm{Zn}, \mathrm{Co}, \mathrm{Mn}, \mathrm{Cr}$}

Hypernova nucleosynthesis may have made an important contribution to Galactic chemical evolution. In the early Galactic epoch when the Galaxy was not yet chemically well-mixed, $[\mathrm{Fe} / \mathrm{H}]$ may well be determined by mostly a single SN event [3]. The formation of metal-poor stars is supposed to be driven by a supernova shock, so that $[\mathrm{Fe} / \mathrm{H}]$ is determined by the ejected $\mathrm{Fe}$ mass and the amount of circumstellar hydrogen swept-up by the shock wave [27]. Then, hypernovae with larger $E$ are likely to induce the formation of stars with smaller $[\mathrm{Fe} / \mathrm{H}]$, because the mass of interstellar hydrogen swept up by a hypernova is roughly proportional to $E[27,30]$ and the ratio of the ejected iron mass to $E$ is smaller for hypernovae than for normal supernovae.

In the observed abundances of halo stars, there are significant differences between the abundance patterns in the iron-peak elements below and above $[\mathrm{Fe} / \mathrm{H}] \sim-2.5--3$.

1. For $[\mathrm{Fe} / \mathrm{H}] \lesssim-2.5$, the mean values of $[\mathrm{Cr} / \mathrm{Fe}]$ and $[\mathrm{Mn} / \mathrm{Fe}]$ decrease toward smaller metallicity, while $[\mathrm{Co} / \mathrm{Fe}]$ increases $[17,27]$.

2. $[\mathrm{Zn} / \mathrm{Fe}] \sim 0$ for $[\mathrm{Fe} / \mathrm{H}] \simeq-3$ to $0[31]$, while at $[\mathrm{Fe} / \mathrm{H}]<-3.3,[\mathrm{Zn} / \mathrm{Fe}]$ increases toward smaller metallicity $[4,26]$.

The larger $[(\mathrm{Zn}, \mathrm{Co}) / \mathrm{Fe}]$ and smaller $[(\mathrm{Mn}, \mathrm{Cr}) / \mathrm{Fe}]$ in the supernova ejecta can be realized if the mass ratio between the complete Si burning region and 

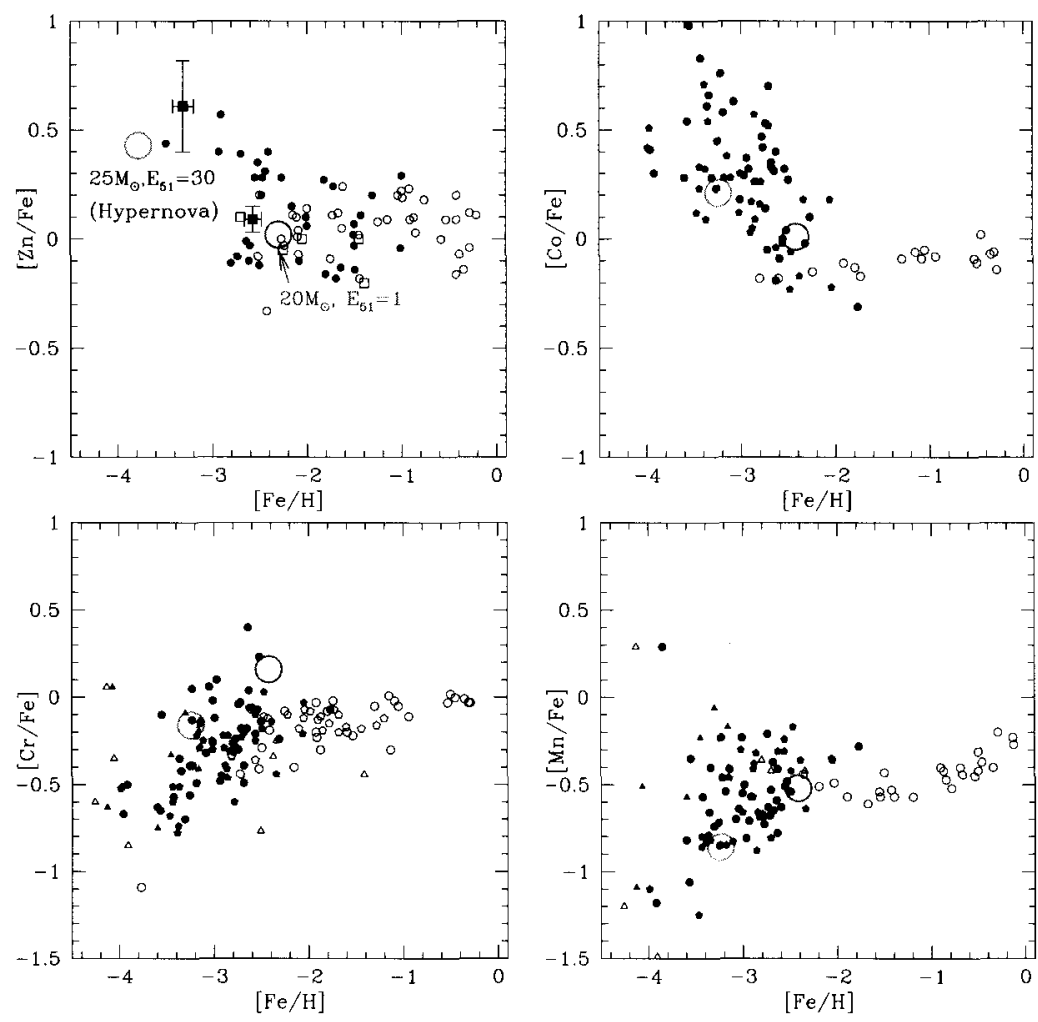

Fig. 3. Observed abundance ratios of $[\mathrm{Zn}, \mathrm{Co}, \mathrm{Cr}, \mathrm{Mn} / \mathrm{Fe}]$ vs $[\mathrm{Fe} / \mathrm{H}]$ compared with $\left(20 \mathrm{M}, E_{51}=1\right)$ and $\left(25 \mathrm{M}, E_{51}=30\right)$ models (large open circles) [36].

the incomplete Si burning region is larger, or equivalently if deep material from the complete Si-burning region is ejected by mixing or aspherical effects. This can be realized if:

1. The mass cut between the ejecta and the compact remnant is located at smaller $M_{r}$ [18];

2. $E$ is larger to move the outer edge of the complete Si burning region to larger $M_{r}[19]$; or

3. Asphericity in the explosion is larger [14].

Among these possibilities, a large explosion energy $E$ enhances $\alpha$-rich freeze out, which results in an increase of the local mass fractions of $\mathrm{Zn}$ and $\mathrm{Co}$, while $\mathrm{Cr}$ and $\mathrm{Mn}$ are not enhanced [25, 33]. Models with $E_{51}=1$ do not produce sufficiently large [Zn/Fe]. To be compatible with the observations of $[\mathrm{Zn} / \mathrm{Fe}] \sim 0.5$, the explosion energy must be much larger, i.e., $E_{51} \gtrsim 20$ for $M \gtrsim 20 \mathrm{M}_{\odot}$, i.e., hypernova-like explosions of massive stars are responsible for the production of $\mathrm{Zn}$. 

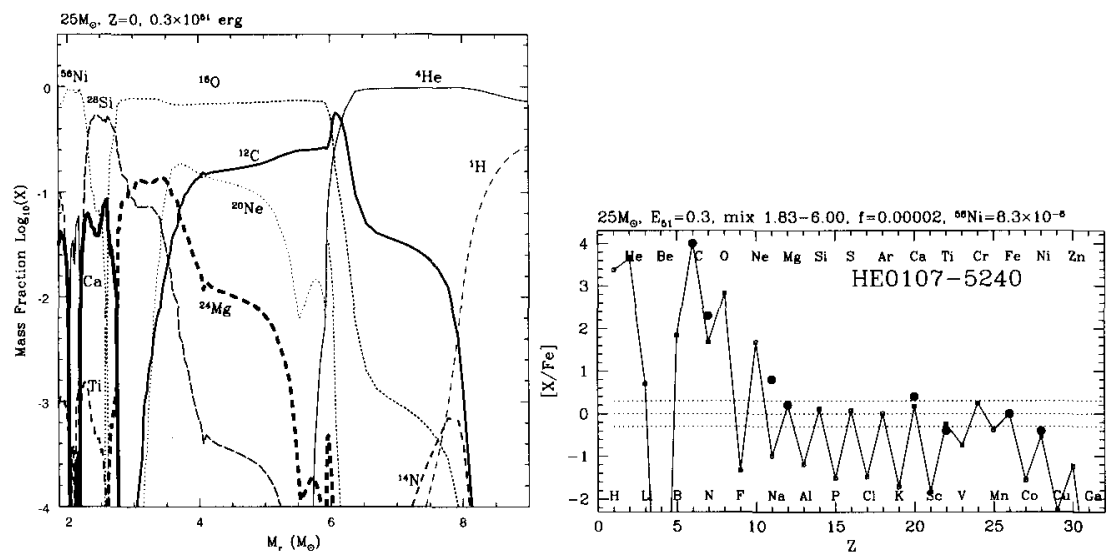

Fig. 4. (left) The post-explosion abundance distributions for the $25 \mathrm{M}_{\odot}$ model with the explosion energy $E_{51}=0.3$ [35]. (right) Elemental abundances of the Crich most Fe deficient star HE0107-5240 (filled circles), compared with a theoretical supernova yield [35].

In the hypernova models, the overproduction of $\mathrm{Ni}$, as found in the simple "deep" mass-cut model, can be avoided [25]. Therefore, if hypernovae made significant contributions to the early Galactic chemical evolution, it could explain the large $\mathrm{Zn}$ and $\mathrm{Co}$ abundances and the small $\mathrm{Mn}$ and $\mathrm{Cr}$ abundances observed in very metal-poor stars (Fig. 3) [36].

\section{Extremely Metal-Poor (EMP) Stars and Faint Supernovae}

Recently the most Fe deficient and C-rich low mass star, HE0107-5240, was discovered [6]. This star has $[\mathrm{Fe} / \mathrm{H}]=-5.3$ but its mass is as low as $0.8 \mathrm{M}_{\odot}$. This would challenge the recent theoretical arguments that the formation of low mass stars, which should survive until today, is suppressed below $[\mathrm{Fe} / \mathrm{H}]$ $=-4[29]$.

The important clue to this problem is the observed abundance pattern of this star. This star is characterized by a very large ratios of $[\mathrm{C} / \mathrm{Fe}]=$ 4.0 and $[\mathrm{N} / \mathrm{Fe}]=2.3$, while the abundances of elements heavier than $\mathrm{Mg}$ are as low as Fe [6]. Interestingly, this is not the only extremely metal poor (EMP) stars that have the large $\mathrm{C} / \mathrm{Fe}$ and $\mathrm{N} / \mathrm{Fe}$ ratios, but several other such stars have been discovered [28]. Therefore the reasonable explanation of the abundance pattern should explain other EMP stars as well. We show that the abundance pattern of C-rich EMP stars can be reasonably explained by the nucleosynthesis of $20-130 \mathrm{M}_{\odot}$ supernovae with various explosion energies and the degree of mixing and fallback of the ejecta. 

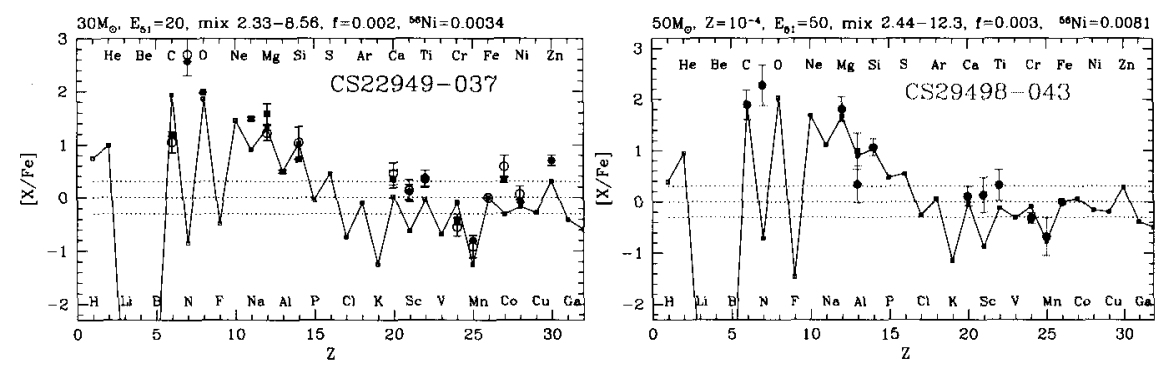

Fig. 5. (left) Elemental abundances of CS 22949-037 (open circles for Norris et al. 2001 [24], and solid squares for Depagne et al. 2002 [7]), compared with a theoretical supernova yield $[35,36]$. (right) Same as the left panel but for CS 29498-043 [2].

\subsection{The Most Fe-poor Star HE0107-5240}

We consider a model that C-rich EMP stars are produced in the ejecta of (almost) metal-free supernova mixed with extremely metal-poor interstellar matter. We use Pop III pre-supernova progenitor models, simulate the supernova explosion and calculate detailed nucleosynthesis [35].

In Fig. 4 (right) we show that the elemental abundances of one of our models are in good agreement with HE0107-5240, where the progenitor mass is $25 \mathrm{M}_{\odot}$ and the explosion energy $E_{51}=0.3$ [35].

In this model, explosive nucleosynthesis takes place behind the shock wave that is generated at $M_{r}=1.8 \mathrm{M}_{\odot}$ and propagates outward. The resultant abundance distribution is seen in Fig. 4 (left), where $M_{r}$ denotes the Lagrangian mass coordinate measured from the center of the pre-supernova model [35]. The processed material is assumed to mix uniformly in the region from $M_{r}=1.8 \mathrm{M}_{\odot}$ and $6.0 \mathrm{M}_{\odot}$. Such a large scale mixing was found to take place in SN1987A and various explosion models [8, 11]. Almost all materials below $M_{r}=6.0 \mathrm{M}_{\odot}$ fall back to the central remnant and only a small fraction $\left(f=2 \times 10^{-5}\right)$ is ejected from this region. The ejected Fe mass is $8 \times 10^{-6}$ $\mathrm{M}_{\odot}$.

The CNO elements in the ejecta were produced by pre-collapse He shell burning in the He-layer, which contains $0.2 \mathrm{M}_{\odot}{ }^{12} \mathrm{C}$. Mixing of $\mathrm{H}$ into the He shell-burning region produces $4 \times 10^{-4} \mathrm{M}_{\odot}{ }^{14} \mathrm{~N}$. On the other hand, only a small amount of heavier elements $(\mathrm{Mg}, \mathrm{Ca}$, and Fe-peak elements) are ejected and their abundance ratios are the average in the region of $M_{r}=1.8-6.0$ $\mathrm{M}_{\odot}$. The sub-solar ratios of $[\mathrm{Ti} / \mathrm{Fe}]=-0.4$ and $[\mathrm{Ni} / \mathrm{Fe}]=-0.4$ are the results of the relatively small explosion energy $\left(E_{51}=0.3\right)$. With this "mixing and fallback", the large $\mathrm{C} / \mathrm{Fe}$ and $\mathrm{C} / \mathrm{Mg}$ ratios observed in HE0107-5240 are well reproduced [35].

In this model, $\mathrm{N} / \mathrm{Fe}$ appears to be under produced. However, $\mathrm{N}$ can be produced inside the EMP stars through the $\mathrm{C}-\mathrm{N}$ cycle, and brought up to the surface during the first dredge up stage while becoming a red-giant star [5]. 

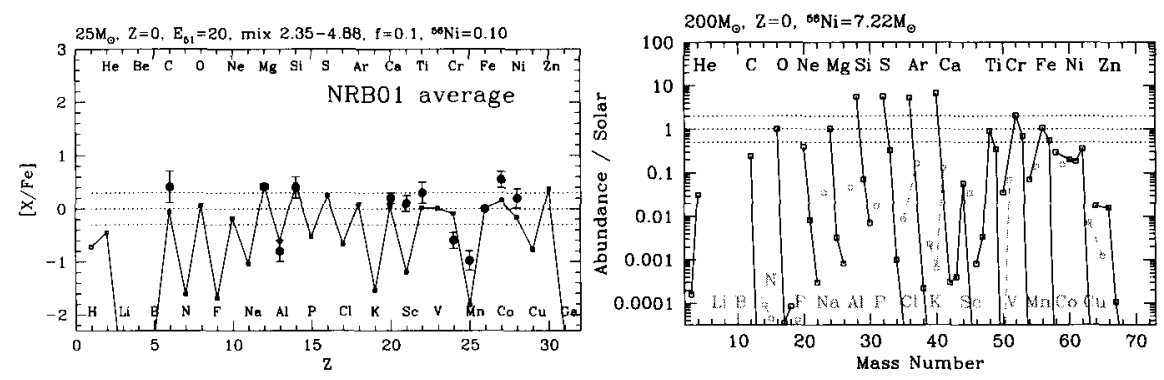

Fig. 6. (left) Averaged elemental abundances of stars with $[\mathrm{Fe} / \mathrm{H}]=-3.7[24]$ compared with a theoretical supernova yield [36]. (right) Yields of a pair-instability supernova from the $200 \mathrm{M}_{\odot}$ star [33].

\subsection{Carbon-rich EMP Stars: CS 22949-037 and CS 29498-043}

The mixing and fallback is commonly required to reproduce the abundance pattern of typical EMP stars. In Fig. 5 (left) we show a model, which is in good agreement with CS22949-037 [35]. This star has $[\mathrm{Fe} / \mathrm{H}]=-4.0$ and also $\mathrm{C}, \mathrm{N}$-rich $[7,24]$, though $\mathrm{C} / \mathrm{Fe}$ and $\mathrm{N} / \mathrm{Fe}$ ratios are smaller than HE0107-5240. The model is the explosion of a $30 \mathrm{M}_{\odot}$ star with $E_{51}=20$. In this model, the mixing region $\left(M_{r}=2.33-8.56 \mathrm{M}_{\odot}\right)$ is chosen to be smaller than the entire He core $\left(M_{r}=13.1 \mathrm{M}_{\odot}\right)$ in order to reproduce relatively large $\mathrm{Mg} / \mathrm{Fe}$ and $\mathrm{Si} / \mathrm{Fe}$ ratios.

Similar degree of the mixing, but for a more massive progenitor, would also reproduce the abundances of CS29498-043 [2], which shows similar abundance pattern (Fig. 5: right),

We assume a larger fraction of ejection than HE0107-5240, $0.2 \%$, from the mixed region for CS22949-037, because the $\mathrm{C} / \mathrm{Fe}$ and $\mathrm{N} / \mathrm{Fe}$ ratios are smaller. The ejected Fe mass is $0.003 \mathrm{M}_{\odot}$. The larger explosion energy model is favored for explaining the large $\mathrm{Zn} / \mathrm{Fe}, \mathrm{Co} / \mathrm{Fe}$ and $\mathrm{Ti} / \mathrm{Fe}$ ratios [33].

Without mixing, elements produced in the deep explosive burning regions, such as $\mathrm{Zn}, \mathrm{Co}$, and $\mathrm{Ti}$, would be under produced. Without fallback the abundance ratios of heavy elements to lighter elements, such as $\mathrm{Fe} / \mathrm{C}, \mathrm{Fe} / \mathrm{O}$, and $\mathrm{Mg} / \mathrm{C}$ would be too large. In this model, $\mathrm{Ti}, \mathrm{Co}, \mathrm{Zn}$ and $\mathrm{Sc}$ are still under produced. However, these elements may be enhanced efficiently in the aspherical explosions $[12,13,14]$.

\subsection{EMP Stars with a Typical Abundance Pattern}

Similarly, the "mixing and fall back" process can reproduce the abundance pattern of the typical EMP stars without enhancement of $\mathrm{C}$ and N. Fig. 6 (left) shows that the averaged abundances of $[\mathrm{Fe} / \mathrm{H}]=-3.7$ stars in Norris et al. [24] can be fitted well with the model of $25 \mathrm{M}_{\odot}$ and $E_{51}=20$ but larger fraction $(\sim 10 \%)$ of the processed materials in the ejecta. This yield [36] is 

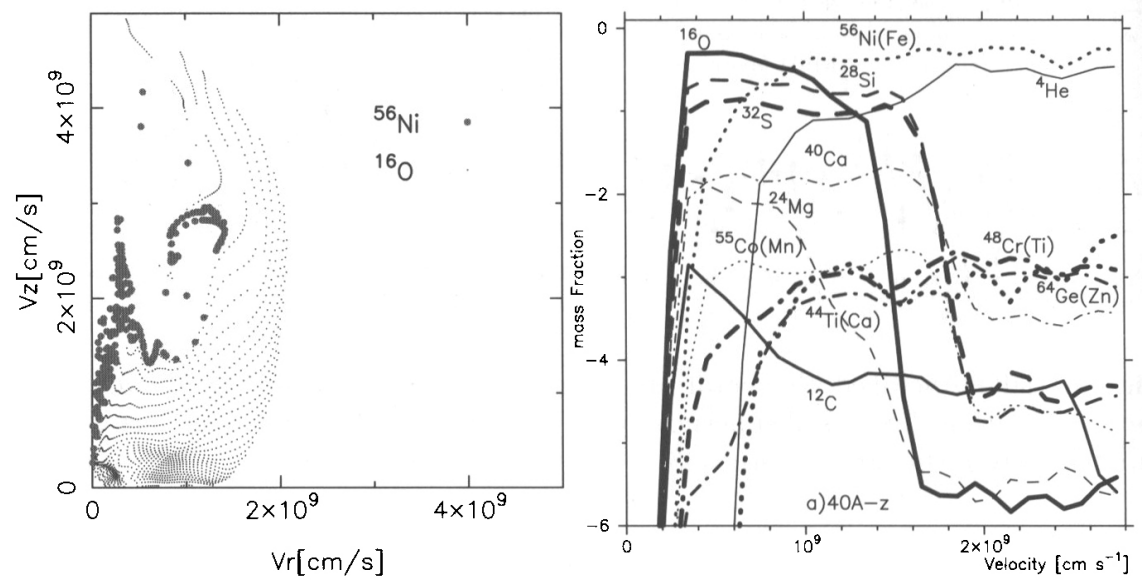

Fig. 7. Left: Distributions of ${ }^{56} \mathrm{Ni}$ (which decays into ${ }^{56} \mathrm{Fe}$ : filled circles) and ${ }^{16} \mathrm{O}$ (dots). The mass elements in which the mass fraction of each isotope exceeds 0.1 are plotted. Right: Mass fractions of selected isotopes in the velocity space along the $z$-axis $[13,14]$.

recommendable as averaged core-collapse $\mathrm{SN}$ yields for the use of chemical evolution models.

\subsection{Aspherical Explosions}

Some observations have indicated that hypernovae ever studied show asphericity to some extent (e.g., [15]). The "mixing and fall-back" effect may also be effectively realized in non-spherical explosions accompanying energetic jets (e.g., $[12,13,14])$. Compared with the spherical model with the same $M_{\text {cut }}(i)$ and $E$, the shock is stronger (weaker) and thus temperatures are higher (lower) in the jet (equatorial) direction. As a result, a larger amount of complete Si-burning products are ejected in the jet direction, while only incomplete Si-burning products are ejected in the equatorial direction (Fig. 7). In total, complete Si-burning elements can be enhanced [13, 14].

The jet-induced explosion results in angle-dependent distribution of nucleosynthetic products as shown in Fig. 7. The distribution of ${ }^{56} \mathrm{Ni}$ (which decays into ${ }^{56} \mathrm{Fe}$ ) is elongated in the jet direction, while that of ${ }^{16} \mathrm{O}$ is concentrated in the central region.

$\mathrm{Zn}$ and $\mathrm{Co}$ are ejected at higher velocities than $\mathrm{Mn}$ and $\mathrm{Cr}$, so that the latter accrete onto the central remnant more easily. As a consequence, $[\mathrm{Zn} / \mathrm{Fe}]$ and $[\mathrm{Co} / \mathrm{Fe}]$ are enhanced, while $[\mathrm{Mn} / \mathrm{Fe}]$ and $[\mathrm{Cr} / \mathrm{Fe}]$ are suppressed. 


\section{The First Stars}

It is of vital importance in current astronomy to identify the first generation stars in the Universe, i.e., totally metal-free, Pop III stars. The impact of the formation of Pop III stars on the evolution of the Universe depends on their typical masses. Recent numerical models have shown that, the first stars are as massive as $\sim 100 \mathrm{M}_{\odot}[1]$. The formation of long-lived low mass Pop III stars may be inefficient because of slow cooling of metal free gas cloud, which is consistent with the failure of attempts to find Pop III stars.

If the most Fe deficient star, HE0107-5240, is a Pop III low mass star that has gained its metal from a companion star or interstellar matter [37], would it mean that the above theoretical arguments are incorrect and that such low mass Pop III stars have not been discovered only because of the difficulty in the observations?

Based on the results in the earlier section, we propose that the first generation supernovae were the explosion of $\sim 20-130 \mathrm{M}_{\odot}$ stars and some of them produced C-rich, Fe-poor ejecta. Then the low mass star with even $[\mathrm{Fe} / \mathrm{H}]$ $<-5$ can form from the gases of mixture of such a supernova ejecta and the (almost) metal-free interstellar matter, because the gases can be efficiently cooled by enhanced $\mathrm{C}$ and $\mathrm{O}([\mathrm{C} / \mathrm{H}] \sim-1)$.

We have shown that the ejecta of core-collapse supernova explosions of $20-130 \mathrm{M}_{\odot}$ stars can well account for the abundance pattern of EMP stars. In contrast, the observed abundance patterns cannot be explained by the explosions of more massive, $130-300 \mathrm{M}_{\odot}$ stars. These stars undergo pairinstability supernovae (PISNe) and are disrupted completely (e.g., [10, 33]), which cannot be consistent with the large C/Fe observed in HE0107-5240 and other C-rich EMP stars. The abundance ratios of iron-peak elements ([Zn/Fe] $<-0.8$ and $[\mathrm{Co} / \mathrm{Fe}]<-0.2$ ) in the PISN ejecta (Fig. 6, $[10,33]$ ) cannot explain the large $\mathrm{Zn} / \mathrm{Fe}$ and $\mathrm{Co} / \mathrm{Fe}$ in the typical EMP stars $[17,24,26]$ and CS22949-037 either. Therefore the supernova progenitors that are responsible for the formation of EMP stars are most likely in the range of $M \sim 20-130$ $\mathrm{M}_{\odot}$, but not more massive than $130 \mathrm{M}_{\odot}$. This upper limit depends on the stability of massive stars.

Acknowledgement. Detailed yields are seen at http://supernova.astron.s.11-tokyo.ac. $\mathrm{jp} / \sim$ umeda/data.html. This work has been supported in part by the grant-in-Aid for COE Scientific Research (14047206, 14540223, 15204010, 16042201, 16540229) of the Ministry of Education, Science, Culture, Sports, and Technology in Japan.

\section{References}

1. T. Abel, G.L. Bryan, M.L. Norman: Science 295, 93 (2002)

2. W. Aoki, S.G. Ryan, T.C. Beers, H. Ando: Astrophys. J. 567, 1166 (2002)

3. J. Audouze, J. Silk: Astrophys. J. Lett. 451, L49 (1995) 
4. L.A.J. Blake, S.G. Ryan, J.E. Norris, T.C. Beers: Nuc. Phys. 688, 502 (2001)

5. A.I. Boothroyd, I.-J. Sackmann: Astrophys. J. 510, 217 (1999)

6. N. Christlieb et al. : Nature 419, 904 (2002)

7. E. Depagne et al. : Astron. Astrophys. 390, 187 (2002)

8. I. Hachisu, T. Matsuda, K. Nomoto, T. Shigeyama: Astrophys. J. Lett. 358, L57 (1990)

9. M. Hashimoto, K. Nomoto, T. Shigeyama: Astron. Astrophys. 210, L5 (1989)

10. A. Heger, S.E. Woosley: Astrophys. J. 567, 532 (2002)

11. K. Kifonidis, T. Plewa, H.-Th. Janka, E. Muller: Astrophys. J. Lett. 531, L123 (2000)

12. K. Maeda, T. Nakamura, K. Nomoto, P.A. Mazzali, F. Patat, I. Hachisu: Astrophys. J. 565, 405 (2002)

13. K. Maeda, K. Nomoto: Nuc. Phys. A718, 167 (2003)

14. K. Maeda, K. Nomoto: Astrophys. J. 598, 1163 (2003)

15. K. Maeda, P.A. Mazzali, J. Deng, K. Nomoto, Y. Yoshii, H. Tomita, Y. Kobayashi: Astrophys. J. 593, 931(2003)

16. P.A. Mazzali, K. Nomoto, J. Deng, K. Maeda, K. Iwamoto, A.V. Filippenko, R.T. Foley: In: these Proceedings

17. A. McWilliam, G.W. Preston, C. Sneden, L. Searle: Astron. J. 109, 2757 (1995)

18. T. Nakamura, H. Umeda, K. Nomoto, F.-K. Thielemann, A. Burrows: Astrophys. J. 517, 193 (1999)

19. T. Nakamura, H. Umeda, K. Iwamoto, K. Nomoto, M. Hashimoto, R.W. Hix, F.-K. Thielemann: Astrophys. J. 555, 880 (2001)

20. K. Nomoto et al. : In: Supernovae and Gamma Ray Bursts, eds. M. Livio et al. (Cambridge Univ. Press: Cambridge, 2001) p. 144

21. K. Nomoto, K. Maeda, H. Umeda, T. Nakamura: In: The Influence of Binaries on Stellar Populations Studies, ed. D. Vanbeveren (Kluwer: Dordrecht, 2001) p. 507

22. K. Nomoto, K. Maeda, H. Umeda, T. Ohkubo, J. Deng, P.A. Mazzali: In: IAU Symp 212, A Massive Star Odyssey, from Main Sequence to Supernova, eds. V.D. Hucht, A. Herrero, C. Esteban (ASP: San Francisco, 2003) p. 395

23. K. Nomoto, K. Maeda, P.A. Mazzali, H. Umeda, J. Deng, K. Iwamoto: astro-ph $0308136(2003)$

24. J.E. Norris, S.G. Ryan, T.C. Beers: Astrophys. J. 561, 1034 (2001)

25. T. Ohkubo, H. Umeda, K. Nomoto: Nuc. Phys. A718, 632 (2003)

26. F. Primas et al. : In: The First Stars, eds. A. Weiss, et al. (Springer: Berlin, 2000) p. 51

27. S.G. Ryan, J.E. Norris, T.C. Beers: Astrophys. J. 471, 254 (1996)

28. S.G. Ryan: astro-ph 0211608 (2002)

29. R. Schneider, A. Ferrara, P. Natarajan, K. Omukai: Astrophys. J. 571, 30 (2002)

30. T. Shigeyama, T. Tsujimoto: Astrophys. J. Lett. 507, L135 (1998)

31. C. Sneden, R.G. Gratton, D.A. Crocker: Astron. Astrophys. 246, 354 (1991)

32. F.-K. Thielemann, K. Nomoto, M. Hashimoto: Astrophys. J. 460, 408 (1996)

33. H. Umeda, K. Nomoto: Astrophys. J. 565, 385 (2002)

34. H. Umeda, K. Nomoto, T. Tsuru, H. Matsumoto: Astrophys. J. 578, 855 (2002)

35. H. Umeda, K. Nomoto: Nature 422, 871 (2003)

36. H. Umeda, K. Nomoto: astro-ph 0308029 (2003)

37. Y. Yoshii: Astron. Astrophys. 97, 280 (1981) 